\title{
CALCULATION OF INDUSTRIAL ENTERPRISE VENTILATION SYSTEM BY NETWORK INTEGRAL METHOD
}

\author{
Evgeniya I. Mihienkova ${ }^{1, *}$, Sergey A. Filimonov ${ }^{2}$, Pavel A. Neobyavlyayuschiy ${ }^{2}$, and \\ Dmitry V. Boykov ${ }^{2}$ \\ ${ }^{1}$ FGAOU VO "Siberian Federal University”, Krasnoyarsk, Russia \\ ${ }^{2}$ OOO “TORINS”, Krasnoyarsk, Russia
}

\begin{abstract}
This paper describe a ventilation system calculation of the technology building industrial enterprise. On the basis of the calculation model for the enterprise offered technical decision of ventilation systems, subject to a compliance exchange multiplicity, purification efficiency, decontamination from the work area; provided the required volume of gas extraction from process equipment according to the sanitary standards and environmental requirements. Produced selection of ventilation equipment parameters, solved the problem of the air exchange balancing between ventilation systems to prevent the emergence of parasitic flows between the rooms building. SigmaNet software package was used for the implement the calculation.
\end{abstract}

\section{Introduction}

Accordance to current sanitary regulations and rules of work site arrangement, both domestic and industrial purposes, in the territory of the Russian Federation must be provided with the optimal parameters of the microclimate. Ventilation is one of the means to ensure the optimum performance of the microclimate. Governing rates for ventilation are air temperature, relative humidity, air velocity in the room and the intensity of the heat generation. At ventilation setting, the calculation appears guarantee of correct functioning system.

Calculation of ventilation systems was carry out by a network integral Theory of hydraulic circuits $[1,2]$, which gives an idea of the balance characteristics studied system (flow, pressure drop, and so on). The hydraulic circuit includes nodes, which provide the mass and energy conservation laws, expressed in the form balance equation and heat energy (Kirchhoff law), and connecting branches, which simulate various designs flue path. Each branch of the network reflects dependence linking the pressure drop at this branch of the gas flow through its cross section.

SigmaNet software package, used for the calculation, designed to simulate the stationary flux-distribution of non-isothermal multicomponent media in the branched pipe installation (ventilation, gas removal). Complex takes into account the chemical reactions in

\footnotetext{
* Corresponding author: mihienkova evgeniya@,mail.ru
} 
the system of pipe installation and heat and mass transfer with the environment. The apparent advantage of this method is a significant reduction in calculation time compared with a computational fluid dynamics calculation, while accuracy preservation.

Initial data for calculation are: project statement of the ventilation system of industrial enterprise building; applicable rules, regulations and requirements.

The ventilation system consists of three independent systems: plenum, general ventilation; process ventilation. Process ventilation ensures the removal, transport and treatment of exhaust gases from the equipment (hoods and ovens), and general ventilation supplies conditioned air to each room and remove the exhaust air from the upper part of the work area to eliminate the local excess concentrations of pollutants.

For correct construction of a network model of ventilation systems built on a threedimensional model of the building scale models of flues in the software package SolidWorks, and was made in the integration of SigmaNet.

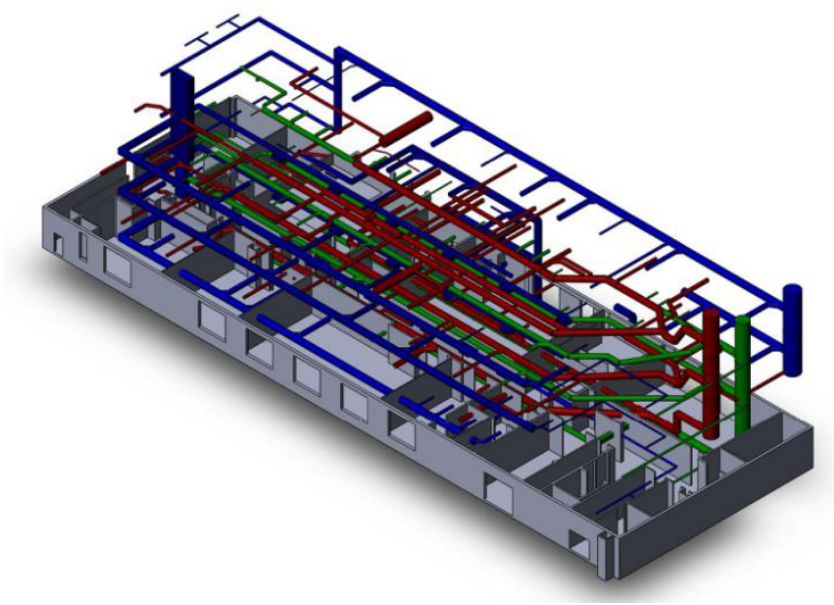

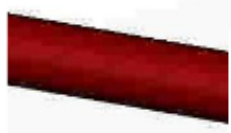

process ventilation

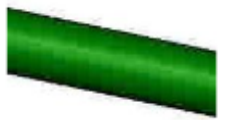

general ventilation

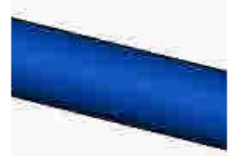

plenum ventilation

Fig. 1. Geometrical model of the flues plant.

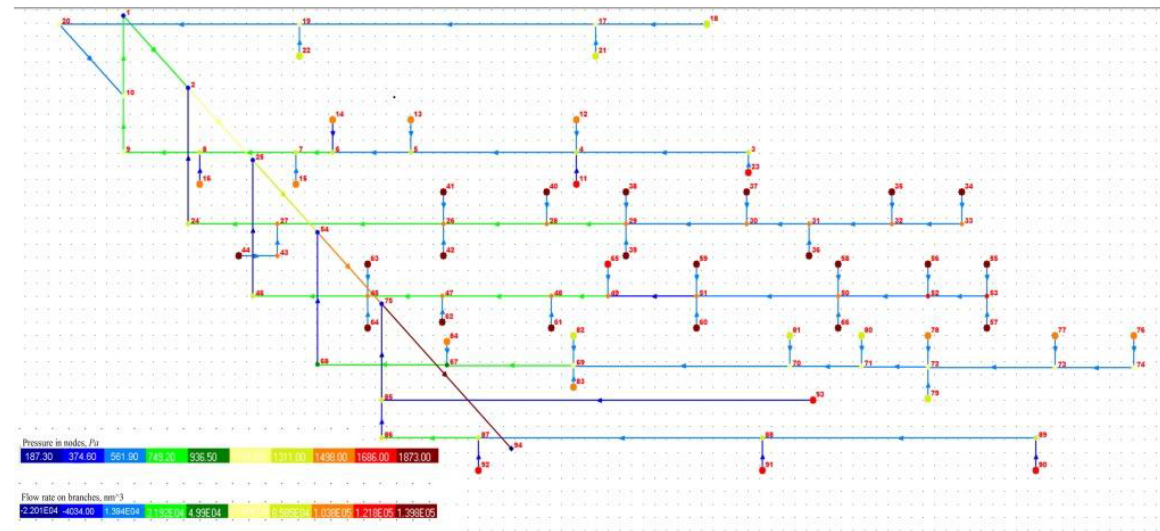

Fig. 2. Calculation of the manufacturing ventilation.

Technical solutions include the use of the general, plenum and process ventilations with dynamic management of operating modes on the technological cycle of the equipment, shift, presence of staff. It is possible to significantly reduce the load on the gas purification and ventilation equipment. Process ventilation collects used air only from working equipment with harmful substances. 


\section{Mathematical model}

Implementation of network methods based on the mathematical model described in paper [1] and thesis [3].

\subsection{Discretization of the continuum motion equations in the network model}

Simulation of non-isothermal multicomponent media reduces to combine problems of flow distribution carrier flow carrier flow, the component and heat flux. At the same time for the relations between the properties of the carrier flow and characteristics of the medium used the equations of state [4].

To represent the network uses a directed graph [5], consisting of two sets: a nodes set $N$ and a branches set (pipes) $U$. Each branch is put in relation pair of nodes, one of which is origin and the other end of this branch (fig. 3-4). Oi is a subset branches starting in $i$-node and $I_{i}$ is subset branches ending at the $i$-node. Also introduced a set internal nodes network $N$-inter, in which the law of conservation.

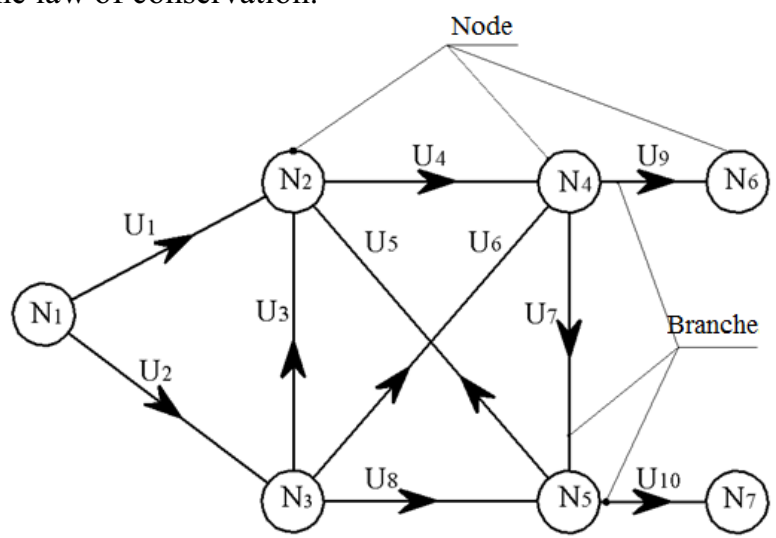

Fig. 3 Graph example.

Set $U_{i}$ for any $i \in N$, is the set branches incident to that node.

The natural orientation of branches $O_{i}\left(u_{l}\right), I_{i}\left(u_{l}\right)$ inserted on the graph to an arbitrarily predetermined flow $u_{l}, l \in U$.

Using expression (1), the flow distribution problem in the network can be reduced to a combination of the law of conservation of mass at the node (2) and in resistance law pipe (3).

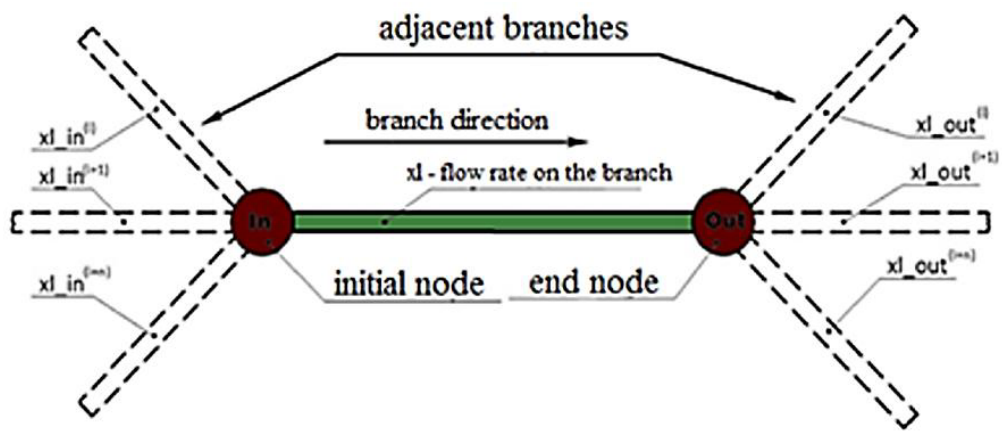

Fig. 4 Sampling network element. 


$$
\begin{gathered}
D_{i l}=\left\{\begin{array}{c}
1, \text { if } l \in O_{i} \\
-1, \text { if } l \in I_{i} \\
0, \text { else }
\end{array}\right. \\
\sum_{l \in U_{i}} D_{i l} x_{l}=q_{i}, i \in N \\
s_{l}\left|x_{l}\right| x_{l}=\sum_{i \in N} D_{i l} \cdot p_{D i}+h_{l}, l \in U
\end{gathered}
$$

$x_{l}$ - mass bearing flow on the branch $(\mathrm{kg} / \mathrm{s}), q_{i}$ - source bearing flow in an existing node $(\mathrm{kg} / \mathrm{s}), p_{D i}$ - node pressure $(\mathrm{Pa}), s_{l}$ - coefficient of resistance, $h_{l}$ - branch head $(\mathrm{Pa})$

\subsection{Hydraulic resistance network site}

Hydraulic resistance is a fundamental value in modeling of the hydraulic circuit. Pressure distribution depends on the correct resistance assignment and flow distribution - in the case of a branched network. Therefore, we consider this value in more detail. According to the manual [6] under the hydraulic resistance (4) refers to total energy (power) loss on a branch to the kinetic energy (power) of the cross-section (for example, at the origin branch), or the ratio of total pressure drop at the branch to dynamic pressure in the cross-section.

$$
\xi_{t o t}=\frac{\Delta P_{t o t}}{2 \cdot \rho \cdot v_{l}^{2}}
$$

$\Delta P_{t o t}$ - total pressure drop the length of branches, $\rho$ - density, $v_{l^{-}}$average flow rate in the branch section.

There are two types of hydraulic resistance (total pressure drop):

Friction resistance $-\xi_{\mathrm{tr}}$. This resistance is due to a hydraulic friction which is caused by viscosity (both molecular and turbulent) real fluids arising in fluid flow.

Local resistance $-\xi_{m}$. The local total pressure loss occur at the local violation of the normal flow, flow separation from the walls, formation of vortex and intensity turbulent mixing in the field of pipeline configuration changes or at a meeting and flow around the obstacles.

Friction resistance (5) at a set subcircuit expresses in terms of the coefficient of hydraulic friction:

$$
\xi_{\text {fr }}=\lambda_{\text {fr }} \frac{l}{d_{\mathrm{g}}}
$$

$\lambda_{\text {fr }}$ - coefficient of flow friction, $d_{\mathrm{g}}$ - hydraulic diameter of branch $(m), l$ - branch length $(m)$.

The coefficient of linear friction depends on the fluid flow conditions and the form of section pipe.

Using the superposition principle of loss in which take the arithmetic sum of friction loss and local loss (6) and formula (4) and (5) we obtain the expression for determining the total pressure drop on the circuit better known as the law of Darcy-Weisbach (7- 8)

$$
\Delta P_{\text {tot }}=\Delta P_{\text {fr }}+\Delta P_{\mathrm{m}}
$$




$$
\Delta P_{\mathrm{tot}}=\left(\frac{\lambda_{\mathrm{fr}} \cdot l}{d_{\mathrm{g}}}+\xi_{\mathrm{m}}\right) \frac{\rho \cdot v_{l}^{2}}{2}
$$

Or going to the symbol in the formula (3):

$$
s_{l}=\left(\frac{\lambda_{\mathrm{fr}} \cdot l}{d_{\mathrm{g}}}+\xi_{\mathrm{m}}\right) \frac{1}{2 \cdot \rho \cdot f^{2}}
$$

$f$ - pipe sectional area $(m)$.

\subsection{Boundary Conditions}

For the numerical scheme is necessary to set the boundary conditions (9), which are set for network with the help of specific types of nodes, namely: "atmospheric" and "source" nodes. The first-order condition for the pressure and concentration of the components set in atmospheric node:

$$
P_{\mathrm{i}}=\text { const } ; \eta_{\mathrm{i}}=\text { const }
$$

Subject to the pressure pattern is relative (system of pressure linear equations is solved up to a constant) in the constructed network model of the hydraulic circuit must be at least one "atmospheric" node.

As for the "source" node, it's characterized by non-zero source / mass flow.

Also worth noting is the following fact: if the node is a border, it does not mean that it is incident with only one branch. However, in the case of the base node number incident branches are 2 or more.

\section{Problem equipment selection}

For the calculation the models of all major elements of ventilation systems create: vertical collectors, gas-main pipeline, lateral pipeline in the room. For correct selection of pumping equipment the necessary flow rate set in each ventilation system for each room. Indoor ventilation network is expressed additional hydraulic resistance on each elements [5].

The following provision was made to ensure the efficiency of hoods: for each lateral pipeline process ventilation, which comes into the room with hoods, set the minimum required pressure.

As a result the calculation of the nodes, simulating rooms, were obtained values of excess pressure / vacuum necessary to provide the required volume of air. At the maximum value of the pressure / vacuum and total flow rate pumping equipment was chosen for each system.

\section{Calculation of ventilation systems}

To align the ventilation systems was developed dynamically balanced model that takes into account the air exchange between the ventilation systems in the rooms and the corridor (fig. 5). In this model, we built all three ventilation system, constraint in between performed using branches, simulating the room (low flow resistance) and branches, simulating the gap between the rooms and the corridor.

For each system boundary condition is pressure from the previous problem. 
Air volumes supplied to the processing space are dynamically balanced with the volume of general and process hoods. On the basis of this task settings controlled damper to regulate the air flow in the building rooms was solved.

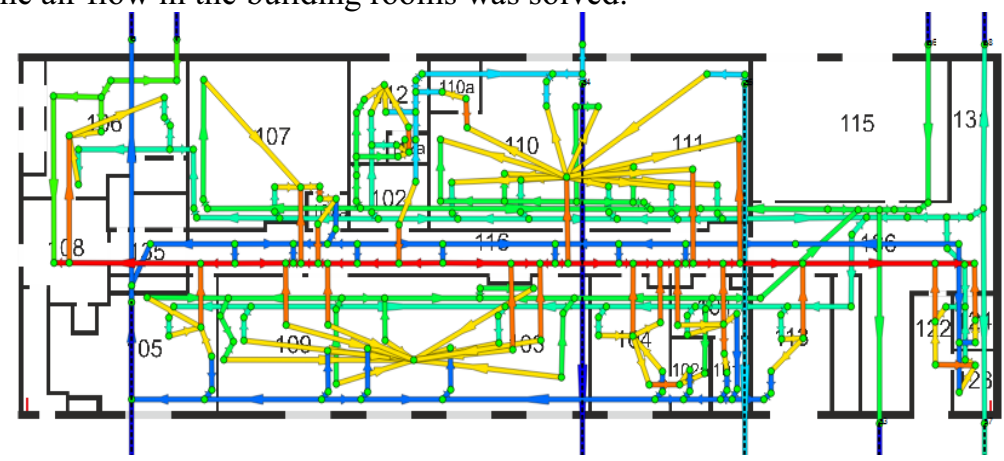

Fig. 5. Model flues 1st floor displaying the network of air exchange between rooms.

The calculations determine the coefficients of local resistance (10) lateral pipeline in the room providing the required flow rate of inflow and hoods. According to the obtained values of local resistance coefficient is determined by the angles of rotation damper. Local resistance coefficient to the rotary shutter was calculated according to the formula [6]:

$$
\xi=\left(\frac{1,56}{1-D^{2} \sin \delta}-1\right)^{2}
$$

$D$ - passing-pipe size $(m), \delta$ - rotation angle of the damper.

\section{Conclusion}

Based on simulation data, we propose technical solutions for ventilation systems in the building enterprise, taking into account the requirements of multiplicity of ventilation, cleaning efficiency, removing hazards from the work area.

A dynamic balanced system of process and general ventilation is developed, which prevents the occurrence of parasitic flows between different enterprise redistributions building.

The flow resistance for each element of the ventilation system calculated, parameters of ventilation equipment chosen.

Rated conditions damper control has been implemented, which provides the necessary air volume for each room.

\section{References}

1. D.V. Boykov, FESTU 38-49, (2006)

2. A.P. Merenkov, V.Ya. Khasilev, Theory of Hydraulic Circuits (Nauka, Moscow 1985) [in Russian]

3. B.A. Elgin, The thesis for the degree of PhD of Physical and Mathematical (1999)

4. S.V. Patankar, Numerical Heat Transfer and Fluid Flow (Hemisphere, 1984)

5. R.Wilson, Introduction to the theory of graphs (Russian transl.: Mir, Moscow, 1977)

6. I.E. Idelchik Manual of hydraulic resistance (Mashinostroenie, Moscow, 1992) [in Russian] 